\title{
SOLVABILITY OF SYSTEMS OF NONHOMOGENEOUS CONVOLUTION EQUATIONS IN CONVEX DOMAINS IN $\mathbb{C}$
}

\author{
A. C. KRIVOSHEEV AND S. N. GANTSEV
}

\begin{abstract}
A criterion for the solvability of systems of nonhomogeneous convolution equations in convex domains on the complex plane is obtained in terms of lower estimates for the characteristic functions of the convolution equations at their noncommon zeros.
\end{abstract}

\section{§1. Introduction. Preliminaries}

Let $D$ be a convex domain in $\mathbb{C}$. We denote by $H(D)$ the space of all functions analytic in $D$, with the topology of uniform convergence on compact subsets of $D$. The space strongly dual to $H(D)$ is denoted by $H^{*}(D)$; its elements are called the analytic functionals in $D$. Since $H(D)$ is a closed subspace of the space of continuous functions in $D$, the theorems of Hahn-Banach and Riesz imply that for each $\mu \in H^{*}(D)$ there exists a measure $m$ with compact support in $D$ such that

$$
(\mu, \psi)=\int_{D} \psi(y) d m(y), \quad \psi \in H(D) .
$$

Roughly, the problem we deal with is as follows. Any functional $\mu$ on the space $H(D)$ gives rise to the operator

$$
M[\psi(z)]=(\mu, \psi(y+z))=\int \psi(y+z) d m(y), \quad \psi \in H(D),
$$

which is called a convolution operator in $H(D)$. Since the operator $M$ is linear and continuous, its values are analytic functions. We can consider an equation of the form

$$
M[\psi(z)]=g, \quad g \in H(G),
$$

where $G$ is a convex domain (to be described below). In this paper we give a criterion for the solvability of systems of such convolution equations with admissible right-hand sides (see below for the definition of admissibility).

A compact convex set $K \subset \mathbb{C}$ is called a determining set for a functional $\mu \in H^{*}(\mathbb{C})$ if for any neighborhood $U$ of $K$ there exists a constant $C_{U}$ such that

$$
|(\mu, \psi)| \leq C_{U} \sup _{z \in U}|\psi(z)|, \quad \psi \in H(\mathbb{C}) .
$$

Let $\mu$ be an analytic functional in $\mathbb{C}$. By the Laplace transform of $\mu$ we mean the function

$$
f(z)=\left(\mu_{y}, \exp (y z)\right), \quad z \in \mathbb{C} .
$$

2000 Mathematics Subject Classification. Primary 45E10, 46E10.

Key words and phrases. Spaces of analytic functions, analytic functional, convolution operator, regular growth.

Supported by the RFBR grant no. 02-01-01100 and by a grant of the President of the Russian Federation for young Doctors of Sciences (no. 00-15-99283). 
This is an entire function of exponential type. Let $K$ be the adjoint diagram of $f$ (see [10]). By the Pólya theorem [10], the set $K$ is the smallest compact convex set determining $\mu$. Since the entire functions are dense in any space $H(\Omega)$, where $\Omega$ is a convex domain in $\mathbb{C}$, inequality (1.2) implies that $\mu$ extends to a continuous linear functional on the space $H(K)$ of analytic functions on $K$. Consequently, if some shift $K+z$ of $K$ lies in a domain $D$, then for any $\psi \in H(D)$ the value of $\mu$ on the element $\psi(y+z), y \in D-z$, of the space $H(D-z) \subset H(K)$ is well defined.

Suppose $D$ has the form $G+K$, where $G$ is a convex domain, and let $\mu \in H^{*}(\mathbb{C})$. The values of the convolution operator (1.1) are analytic functions in $G$, i.e., $M$ acts from $H(D)$ to $H(G)$. The Laplace transform $f$ of the functional $\mu$ is called the characteristic function of the operator $M$.

Let $G$ be a convex domain in $\mathbb{C}$, let $\mu_{1}, \ldots, \mu_{n}$ be analytic functionals in $\mathbb{C}$, and let $f_{1}, \ldots, f_{n}$ and $K_{1}, \ldots, K_{n}$ be the corresponding Laplace transforms and adjoint diagrams, respectively. We put

$$
D=G+K_{1}+\cdots+K_{n}, \quad G_{i}=G+K_{1}+\cdots+K_{i-1}+K_{i+1}+\cdots+K_{n}, \quad i=1, \ldots, n .
$$

Then for each $i=1, \ldots, n$, the functional $\mu_{i}$ determines a convolution operator $M_{i}$ acting from $H(D)$ to $H\left(G_{i}\right)$. Consider the following system of convolution equations:

$$
M_{1}[\psi]=g_{1}, \ldots, M_{n}[\psi]=g_{n} .
$$

The question about solvability conditions for this system arises naturally. For $n=1$, i.e., for one convolution equation, there are numerous publications devoted to this solvability problem; a complete solution was presented in 3 , 4. for the case of convex domains in the complex space of arbitrary dimension. For smooth domains, full solution of a system of nonhomogeneous convolution equations was proposed in the paper [5], where there is also a list of the most important references on the topic.

We dwell on the results of [5] in more detail. The notion of regular growth for a system of entire functions was introduced in [5]. This is a generalization of the classical notion of complete regularity of the growth for an individual entire function. The definition in [5] involves lower estimates of the maximal absolute values of $f_{1}, \ldots, f_{n}$ on a set of open disks. On that basis, sufficient conditions were obtained for the solvability of a system of convolution equations in convex domains in $\mathbb{C}$. Moreover, it was shown that in a number of cases these conditions are also necessary, which provided a solvability criterion for (1.3). For instance, if the boundary of $G$ is smooth, then the solvability of (1.3) is equivalent to the fact that the system $\left(f_{1}, \ldots, f_{n}\right)$ has regular growth. The proof of sufficiency in [5] was based on complicated induction constructions and on the solvability of cohomology groups with estimates.

In the present paper we establish some conditions equivalent to the fact that a system of entire functions has regular growth in the sense of [5]. These new conditions are given in terms of lower estimates for the behavior of the functions $f_{1}, \ldots, f_{n}$ at their noncommon zeros. Unlike the method used in [5], our arguments here do not involve induction constructions and are substantially simpler. This method combines the approach that goes back to Wolff (see [6, Appendix]) with some ideas used in [5].

We start with some known facts and notions needed in what follows. Suppose the domains $D, G$, and $G_{i}$, the functionals $\mu_{i}$, the functions $f_{i}$, and the operators $M_{i}$ are as described above. We put $M=\left(M_{1}, \ldots, M_{n}\right)$ and

$$
M[\psi]=\left(M_{1}[\psi], \ldots, M_{n}[\psi]\right) .
$$

The operator $M$ acts from $H(D)$ to the product $H=H\left(G_{1}\right) \times \cdots \times H\left(G_{n}\right)$. Let $M^{*}$ be the operator adjoint to $M$, acting from $H^{*}\left(G_{1}\right) \times \cdots \times H^{*}\left(G_{n}\right)$ to $H^{*}(D)$, and let $P_{D}$ denote the space of entire functions of exponential type the adjoint diagrams of which 
lie in $D$. The topology in $P_{D}$ is defined as that of the direct limit of the Banach spaces

$$
P_{j}=\left\{\varphi \in H(\mathbb{C}):\|\varphi\|_{j}=\sup _{z \in \mathbb{C}}|\varphi(z)| \exp \left[-H_{L_{j}}(z)\right]<+\infty\right\}
$$

where the $L_{j}$ constitute a sequence of compact convex subsets of $D$ such that $L_{j} \subset$ $\operatorname{int} L_{j+1}$ and $\bigcup L_{j}=D$, and $H_{L}$ denotes the support function of a set $L$ :

$$
H_{L}(y)=\sup _{z \in L} \operatorname{Re}(z y), \quad y \in \mathbb{C} .
$$

Since the embeddings are compact, the definition of the topology in $P_{D}$ implies (see, e.g., [7]) that a sequence $\left\{\varphi_{k}\right\} \subset P_{D}$ converges in $P_{D}$ to a function $\varphi$ if $\varphi$ and $\left\{\varphi_{k}\right\}$ belong to $P_{j}$ for some $j$ and $\left\{\varphi_{k}\right\}$ tends to $\varphi$ in $P_{j}$. In its turn, convergence in $P_{j}$ means that for some $C>0$ and all $k$ we have $\left|\varphi_{k}(z)\right| \leq C \exp H_{L_{j}}(z), z \in \mathbb{C}$, and $\left\{\varphi_{k}\right\}$ tends to $\varphi$ uniformly on every compact set in $\mathbb{C}$.

It is well known that the Laplace transformation establishes an algebraic as well as topological isomorphism of the spaces $H^{*}(D)$ and $P_{D}$ (see 7]); consequently, $M^{*}$ determines an operator $\Sigma$ acting from $P_{G_{1}} \times \cdots \times P_{G_{n}}$ to $P_{D}$. Let $\nu=\left(\nu_{1}, \ldots, \nu_{n}\right) \in$ $H^{*}\left(G_{1}\right) \times \cdots \times H^{*}\left(G_{n}\right)$, and let $\mu=M^{*}[\nu]$. We denote by $\varphi_{i}$ and $F$ the Laplace transforms of $\nu_{i}$ and $\mu$, respectively. For calculating the operator $\Sigma$ explicitly we plug the exponential function in the identity that defines the adjoint operator, obtaining

$$
\begin{aligned}
F(z) & =\left(M^{*}[\nu], \exp (z \xi)\right)=(M[\exp (z \xi)], \nu)=\sum_{i=1}^{n}\left(\left(\mu_{i}, \exp ((y+\xi) z)\right), \nu_{i}\right) \\
& =\sum_{i=1}^{n}\left(\left(\mu_{i}, \exp (z \xi)\right) \exp (y z), \nu_{i}\right)=\sum_{i=1}^{n} f_{i}(z)\left(\exp (y z), \nu_{i}\right)=\sum_{i=1}^{n} f_{i}(z) \varphi_{i}(z) .
\end{aligned}
$$

Thus, for each vector $\varphi=\left(\varphi_{1}, \ldots, \varphi_{n}\right)$ in $P\left(G_{1}\right) \times \cdots \times P\left(G_{n}\right)$, we have

$$
\Sigma[\varphi](z)=f_{1}(z) \varphi_{1}(z)+\cdots+f_{n}(z) \varphi_{n}(z) .
$$

Suppose a function $g \in H$ lies in the range of $M$, i.e., $M[h]=g$ for some $h \in H(D)$. If $\nu \in \operatorname{ker} M^{*}$, then

$$
(\nu, g)=(\nu, M[h])=\left(M^{*}[\nu], h\right)=0 .
$$

Consequently, system (1.3) with the right-hand side $g=\left(g_{1}, \ldots, g_{n}\right)$ can be solvable only if $(\nu, g)=0$ for any $\nu \in \operatorname{ker} M^{*}$. Recalling the explicit form of the operator $\Sigma$, we can rewrite this condition in terms of Laplace transforms as follows: $(\nu, g)=0$ for every $\nu=\nu_{1}, \ldots, \nu_{n}$ such that $f_{1} \varphi_{1}+\cdots+f_{n} \varphi_{n}=0\left(\varphi_{1}, \ldots, \varphi_{n}\right.$ are as before $)$. We say that a right-hand side $g$ of system (1.3) is admissible if it satisfies the above condition. Obviously, the set of all admissible right-hand sides is a closed subspace in $H$. Thus, the question as to whether system (1.3) is solvable makes sense only for elements of that subspace.

Lemma 1.1 ([5]). The range of the operator $M$ is dense in the set of admissible righthand sides of system (1.3).

This lemma implies that system (1.3) is solvable in $H(D)$ for any admissible right-hand side $g \in H$ if and only if the range of $M$ is closed. Since $H(D)$ and $H\left(G_{i}\right)$ are complete metrizable spaces, i.e., they are Frechét spaces, the theorem on adjoint operation for such spaces (see [7]) shows that the range im $M$ is closed if and only if the range of the adjoint operator $M^{*}$ is closed, or, equivalently, the set im $\Sigma \subset P_{D}$ is closed.

Let $f_{1}, \ldots, f_{n}$ be the Laplace transforms of functionals $\mu_{1}, \ldots, \mu_{n}$, and let $\Lambda=\left\{\lambda_{k}\right\}$ be the sequence of common zeros of $f_{1}, \ldots, f_{n}$ (with regard to multiplicity, i.e., if $\lambda$ is a common zero of the functions $f_{1}, \ldots, f_{n}$ with multiplicity $m$, then $\lambda$ occurs $m$ times in 
the sequence $\Lambda)$. We define $I(D, \Lambda)$ as the set of all functions $\psi \in P_{D}$ such that $\Lambda$ is part of the zeros of $\psi$.

We conclude this section with a lemma that reduces the solvability problem for system (1.3) to the problem of a certain specific representation of the elements of the subspace $I(D, \Lambda)$.

Lemma $1.2([5])$. The following two statements are equivalent.

1) For any $F \in I(D, \Lambda)$, there is an element $\left(\varphi_{1}, \ldots, \varphi_{n}\right) \in P_{G_{1}} \times \cdots \times P_{G_{n}}$ such that

$$
F(z)=\varphi_{1}(z) f_{1}(z)+\cdots+\varphi_{n}(z) f_{n}(z), \quad z \in \mathbb{C} .
$$

2) The system of convolution equations (1.3) is solvable in the space $H(D)$ for any admissible right-hand side $g \in H$.

\section{§2. Regular GROWTH OF A SYSTEM OF ENTIRE FUNCTIONS OF FINITE ORDER AND TYPE}

The notion of regular and weakly regular growth for a system of entire functions of order (at most) $\rho$ and of finite type (with order $\rho$ ) was introduced in [5]. Here we give an equivalent definition; unlike the one in [5], this definition is based on the mutual location of noncommon zeros of the system of entire functions in question.

Consider $n$ collections of complex numbers

$$
\left(a_{1}^{1}, a_{2}^{1}, \ldots, a_{k_{1}}^{1}\right), \quad\left(a_{1}^{2}, a_{2}^{2}, \ldots, a_{k_{2}}^{2}\right), \quad \ldots, \quad\left(a_{1}^{n}, a_{2}^{n}, \ldots, a_{k_{n}}^{n}\right) .
$$

For each of these collections, we construct the following polynomial:

$$
P_{i}(z)=b_{i} \prod_{j=1}^{k_{i}}\left(z-a_{j}^{i}\right), \quad z \in \mathbb{C},
$$

where $\left\{b_{i}\right\} \in \mathbb{C} \backslash\{0\}$.

Theorem 2.1. Suppose the inequalities

$$
\max _{1 \leq i \leq n} \ln \left|P_{i}\left(a_{l}^{p}\right)\right| \geq-B, \quad 1 \leq p \leq n, \quad 1 \leq l \leq k_{p},
$$

are fulfilled for some $B>0$. Then for all $z \in \mathbb{C}$ we have

$$
\max _{1 \leq i \leq n}\left[\ln \left|P_{i}(z)\right|+k_{i} \ln 2\right] \geq-B .
$$

Proof. By the assumptions of the theorem, for every $p=1, \ldots, n$ and every $l=1, \ldots, k_{p}$ there exists an index $i_{p, l}$ such that

$$
\ln \left|P_{i_{p, l}}\left(a_{l}^{p}\right)\right| \geq-B .
$$

Let $B(x, r)$ denote the open disk of radius $r$ and centered at $x$. It is easily seen that for all $z \notin B\left(a_{j}^{i_{p, l}},\left|a_{l}^{p}-a_{j}^{i_{p, l}}\right| / 2\right)$ we have the estimate $2\left|z-a_{j}^{i_{p, l}}\right| \geq\left|a_{l}^{p}-a_{j}^{i_{p, l}}\right|$. The last two inequalities imply that outside the union $\bigcup_{j=1}^{k_{i_{p}, l}} B\left(a_{j}^{i_{p, l}},\left|a_{l}^{p}-a_{j}^{i_{p, l}}\right| / 2\right)$ the following estimate is true::

$$
\ln \left|P_{i_{p, l}}(z)\right|+k_{i_{p, l}} \ln 2 \geq-B .
$$

Uniting these estimates for all $p=1, \ldots, n$ and $l=1, \ldots, k_{p}$, we see that for any $z$ off the set $\Omega=\bigcap_{p=1}^{n} \bigcap_{l=1}^{k_{p}} \bigcup_{j=1}^{k_{i}, l} B\left(a_{j}^{i_{p, l}},\left|a_{l}^{p}-a_{j}^{i_{p, l}}\right| / 2\right)$ we have

$$
\max _{1 \leq i \leq n}\left[\ln \left|P_{i}(z)\right|+k_{i} \ln 2\right] \geq \max _{\substack{1 \leq p \leq n \\ 1 \leq l \leq k_{p}}}\left[\ln \left|P_{i_{p, l}}(z)\right|+k_{i_{p, l}} \ln 2\right] \geq-B .
$$

The theorem will be proved if we show that $\Omega=\varnothing$. 
Suppose $z \in \Omega$. Then for each $p=1, \ldots, n$ and each $l=1, \ldots, k_{p}$, there is an index $j(p, l, z)$ such that $z$ belongs to the disk $B\left(a_{j(p, l, z)}^{i_{p, l}},\left|a_{l}^{p}-a_{j(p, l, z)}^{i_{p, l}}\right| / 2\right)$. But then $z$ will also belong to the set

$$
\Omega(z)=\bigcap_{p=1}^{n} \bigcap_{l=1}^{k_{p}} B\left(a_{j(p, l, z)}^{i_{p, l}},\left|a_{l}^{p}-a_{j(p, l, z)}^{i_{p, l}}\right| / 2\right) .
$$

We shall show that the set $\Omega(z)$ is empty for every $z \in \Omega$. Assume the contrary, i.e., there exists $z \in \Omega$ such that $\Omega(z) \neq \varnothing$.

Let $B\left(a_{j_{2}}^{i_{2}},\left|a_{j_{1}}^{i_{1}}-a_{j_{2}}^{i_{2}}\right| / 2\right)$ be one of the disks occurring in (2.1). There is an index $j_{3}$ such that the disk $B\left(a_{j_{3}}^{i_{3}},\left|a_{j_{2}}^{i_{2}}-a_{j_{3}}^{i_{3}}\right| / 2\right)$ occurs in the same intersection (2.1). This is true because $p$ runs over all values from 1 to $n$, and $l$ ranges from 1 to $k_{p}$. Our assumption implies that

$$
\left|a_{j_{1}}^{i_{1}}-a_{j_{2}}^{i_{2}}\right|>\left|a_{j_{2}}^{i_{2}}-a_{j_{3}}^{i_{3}}\right| .
$$

In a similar way, for the disk $B\left(a_{j_{3}}^{i_{3}},\left|a_{j_{2}}^{i_{2}}-a_{j_{3}}^{i_{3}}\right| / 2\right)$ we can find a disk $B\left(a_{j_{4}}^{i_{4}},\left|a_{j_{3}}^{i_{3}}-a_{j_{4}}^{i_{4}}\right| / 2\right)$ with

$$
\left|a_{j_{1}}^{i_{1}}-a_{j_{2}}^{i_{2}}\right|>\left|a_{j_{2}}^{i_{2}}-a_{j_{3}}^{i_{3}}\right|>\left|a_{j_{3}}^{i_{3}}-a_{j_{4}}^{i_{4}}\right|
$$

Continuing, at the $k$ th step we obtain

$$
\left|a_{j_{1}}^{i_{1}}-a_{j_{2}}^{i_{2}}\right|>\cdots>\left|a_{j_{k-1}}^{i_{k-1}}-a_{j_{k}}^{i_{k}}\right|>\left|a_{j_{k}}^{i_{k}}-a_{j_{k+1}}^{i_{k+1}}\right| .
$$

This procedure can be done as many times as we wish. Since the number of disks occurring in (2.1) is finite, at some step there will be an index $m_{1}$ such that

$$
\left|a_{j_{1}}^{i_{1}}-a_{j_{2}}^{i_{2}}\right|>\cdots>\left|a_{j_{m_{1}}}^{i_{m_{1}}}-a_{j_{m_{1}+1}}^{i_{m_{1}+1}}\right|>\left|a_{j_{m_{1}+1}}^{i_{m_{1}+1}}-a_{j_{m_{1}+2}}^{i_{m_{1}+2}}\right|>\cdots>\left|a_{j_{m_{1}}}^{i_{m_{1}}}-a_{j_{m_{1}+1}}^{i_{m_{1}+1}}\right|>\cdots,
$$

a contradiction. Thus, the set $\Omega(z)$ is empty for any $z \in \Omega$, which implies that $\Omega=\varnothing$. The theorem is proved.

Let $\psi(z)$ be an entire function of order (at most) $\rho>0$ and of finite type (with order $\rho$ ), i.e., for some $a, b>0$ we have the inequality

$$
|\psi(z)| \leq a \exp \left(b|z|^{\rho}\right), \quad z \in \mathbb{C} .
$$

We denote by $h_{\psi}(z)$ the indicator of the function $\psi(z)$ :

$$
h_{\psi}(z)=\varlimsup_{t \rightarrow+\infty} \ln |\psi(t z)| / t^{\rho}, \quad z \in \mathbb{C} .
$$

The function $h_{\psi}(z)$ is subharmonic, positively homogeneous of order $\rho$ (i.e., $h_{\psi}(t z)=$ $t^{\rho} h_{\psi}(z), z \in \mathbb{C}, t \geq 0$ ), and continuous on $\mathbb{C}$ (see [2]).

Let $\mathbf{S}$ be the unit circle centered at zero, and let $z \in \mathbf{S}$. For $\delta>0$, we denote by $E(z, \delta)$ the collection of all sequences $\left\{z_{k}\right\}_{k=1}^{\infty}$ of points on $\{t z, t>0\}$ that satisfy $\left|z_{k}\right| \rightarrow+\infty$ and

$$
\left|z_{k+1}\right| /\left|z_{k}\right|<1+\delta, \quad k=1,2, \ldots
$$

Next, let $E(z)$ be the set of sequences $\left\{z_{k}\right\}_{k=1}^{\infty}$ that lie on $\{t z, t>0\}$ and satisfy the following condition: for every $\delta>0$ and some $k(\delta)$, the sequence $\left\{z_{k}\right\}_{k=k(\delta)}^{\infty}$ is contained in $E(z, \delta)$.

Let $\Lambda=\left\{\lambda_{j}\right\}_{j=1}^{\infty} \subset \mathbb{C}$. For every $\delta>0$ and every $0 \neq x \in \mathbb{C} \backslash \Lambda$, we introduce the functions

$$
\begin{aligned}
& q_{\delta}(z, x, \Lambda)=\prod_{\lambda_{j} \in B(x, \delta|x|)}\left(z-\lambda_{j}\right) /\left(x-\lambda_{j}\right), \\
& \widetilde{q}_{\delta}(z, x, \Lambda)=\prod_{\lambda_{j} \in B(x, \delta|x|)}\left(z-\lambda_{j}\right) /(2 \delta x), \quad z \in \mathbb{C} .
\end{aligned}
$$


In the case where $B(x, \delta|x|)$ contains no points of the sequence $\left\{\lambda_{j}\right\}$, we put $q_{\delta}(z, x, \Lambda) \equiv 1$ and $\widetilde{q}_{\delta}(z, x, \Lambda) \equiv 1$. The following relations can be verified easily:

$$
\begin{aligned}
q_{\delta}(x, x, \Lambda) & =1 ; & & \\
\left|q_{\delta}(z, x, \Lambda)\right| & \geq 1, & & z \notin B(x, 2 \delta|x|) ; \\
\left|\widetilde{q}_{\delta}(z, x, \Lambda)\right| & <1, & & z \in B(x, \delta|x|) ; \\
\left|\widetilde{q}_{\delta}(z, x, \Lambda)\right| & \geq 1, & & z \notin B(x, 3 \delta|x|) ; \\
\left|q_{\delta}(z, x, \Lambda)\right| & >\left|\widetilde{q}_{\delta}(z, x, \Lambda)\right|, & & z \in \mathbb{C} .
\end{aligned}
$$

Let $\Theta_{i}$ be the set of all zeros of $\psi_{i}$, and let $\Lambda=\left\{\lambda_{j}\right\}_{j=1}^{\infty}$ be the sequence of all common zeros of the system $\left(\psi_{1}, \ldots, \psi_{n}\right)$ (with regard to multiplicity). We put $\Lambda_{i}=\left\{\lambda_{j}^{i}\right\}=\Theta_{i} \backslash \Lambda$ and call $\Lambda_{i}$ the set of noncommon zeros of the function $\psi_{i}$.

Definition 1 ([5]). We say that a system $\left(\psi_{1}, \ldots, \psi_{n}\right)$ of entire functions of order (at most) $\rho$ and of finite type (with order $\rho$ ) has regular growth along a ray ty $(y \in \mathbf{S}$ ) if there exists a sequence $\left\{z_{k}\right\} \in E(y)$ satisfying the following condition: for any $r \in(0,1)$ and any $\varepsilon>0$, there is $\delta(r, \varepsilon)>0$ and an index $k_{0}$ such that the inequalities

$$
\max _{1 \leq i \leq n}\left[\ln \left|\frac{\psi_{i}(z)}{q_{\delta}\left(z, z_{k}, \Lambda\right)}\right|-h_{\psi_{i}}(z)\right] \geq-\varepsilon|z|^{\rho}, \quad z \in B\left(z_{k}, r \delta\left|z_{k}\right|\right), \quad k \geq k_{0},
$$

are fulfilled for every $\delta \in(0, \delta(r, \varepsilon))$, where $\Lambda=\left\{\lambda_{j}\right\}_{j=1}^{\infty}$ is as above.

Now, on the basis of this definition and Theorem[2.1, we give the following definition of regular growth for a system of functions and prove that it is equivalent to Definition 1.

Definition 2. We say that a system $\left(\psi_{1}, \ldots, \psi_{n}\right)$ of entire functions of order (at most) $\rho$ and of finite type (with order $\rho$ ) has regular growth along a ray ty $(y \in \mathbf{S}$ ) if there exists a sequence $\left\{z_{k}\right\} \in E(y)$ with the following property: for any $r \in(0,1)$ and any $\varepsilon>0$, there is $\delta(r, \varepsilon)>0$ and an index $k_{0}$ such that the next two conditions are fulfilled for every $\delta \in(0, \delta(r, \varepsilon))$ :

1) $\max _{1 \leq i \leq n}\left[\ln \left|\psi_{i}\left(z_{k}\right)\right|-h_{\psi_{i}}\left(z_{k}\right)\right] \geq-\varepsilon\left|z_{k}\right|^{\rho}, k \geq k_{0}$;

2) for any $1 \leq p \leq n$ and all $\lambda_{l}^{p} \in B\left(z_{k}, r \delta\left|z_{k}\right|\right)$ we have

$$
\max _{1 \leq i \leq n}\left[\ln \left|\frac{\psi_{i}\left(\lambda_{l}^{p}\right)}{q_{\delta}\left(\lambda_{l}^{p}, z_{k}, \Lambda\right)}\right|-h_{\psi_{i}}\left(\lambda_{l}^{p}\right)\right] \geq-\varepsilon\left|\lambda_{l}^{p}\right|^{\rho}, \quad k \geq k_{0} .
$$

Unlike Definition 1, in Definition 2 a lower estimate is required not everywhere in the disk $B\left(z_{k}, r \delta\left|z_{k}\right|\right)$, but only at the noncommon zeros of each of the functions $\psi_{i}, i=$ $1, \ldots, n$. As to condition 1) in Definition 2, it is in fact required only in the case where no function $\psi_{i}, i=1, \ldots, n$, has noncommon zeros in the disk $B\left(z_{k}, r \delta\left|z_{k}\right|\right)$.

Proposition 1. Definitions 1 and 2 are equivalent.

Proof. Obviously, Definition 1 implies Definition 2, We prove the converse. Suppose there exists a sequence $\left\{z_{k}\right\} \in E(y)$ with the following property: for any $\widetilde{r} \in(0,1)$ and any $\widetilde{\varepsilon}>0$ we can find $\delta(\widetilde{r}, \widetilde{\varepsilon})>0$ and an index $k_{1}$ such that the requirements of Definition 2 are fulfilled for every $\delta \in(0, \delta(\widetilde{r}, \widetilde{\varepsilon}))$.

From the continuity of the indicator $h_{\psi_{i}}(z)$, and from the Hartogs theorem on families of subharmonic functions it is easy to deduce (see, e.g., 1]) the existence of numbers $R, \delta^{\prime}>0$ such that

$$
\ln \left|\psi_{i}(t z)\right| / t^{\rho} \leq h_{\psi_{i}}(y)+\widetilde{\varepsilon}, \quad z \in B\left(y, 12 \delta^{\prime}\right), \quad t>R .
$$

Since $h_{\psi_{i}}(z)$ is continuous, we may assume (reducing $\delta^{\prime}>0$ if necessary) that

$$
\left|h_{\psi_{i}}(y)-h_{\psi_{i}}(z)\right|<\widetilde{\varepsilon}, \quad z \in B\left(y, \delta^{\prime}\right) .
$$


By condition 1) of Definition 2, there is an index $k_{2}$ such that $\left|z_{k}\right|>R$ and

$$
\max _{1 \leq i \leq n}\left[\ln \left|\psi_{i}\left(z_{k}\right)\right| /\left|z_{k}\right|^{\rho}-h_{\psi_{i}}(y)\right] \geq-\widetilde{\varepsilon}, \quad k \geq k_{2} .
$$

For $k \geq k_{2}$, we consider the functions

$$
\begin{aligned}
& \varphi_{i, k}(z)=\psi_{i}(z)\left[\widetilde{q}_{\widetilde{r} \delta}\left(z, z_{k}, \Lambda_{i}\right) q_{\delta}\left(z, z_{k}, \Lambda\right)\right]^{-1}, \quad \delta \in\left(0, \min \left(\delta^{\prime}, \delta(\widetilde{r}, \widetilde{\varepsilon})\right) .\right. \\
& \widetilde{\varphi}_{i, k}(z)=\psi_{i}(z) / q_{\delta}\left(z, z_{k}, \Lambda\right),
\end{aligned}
$$

By construction, the $\varphi_{i, k}$ and $\widetilde{\varphi}_{i, k}$ are entire functions. It is easily seen that $\widetilde{\varphi}_{i, k}\left(z_{k}\right)=$ $\psi_{i}\left(z_{k}\right)$.

Consider the case where the functions $\psi_{i}, i=1, \ldots, n$, have no noncommon zeros in $B\left(z_{k}, \widetilde{r} \delta\left|z_{k}\right|\right)$. By (2.5), for every $k \geq k_{2}$ there is an index $i(k)$ such that

$$
\ln \left|\psi_{i(k)}\left(z_{k}\right)\right| /\left|z_{k}\right|^{\rho}-h_{\psi_{i(k)}}(y) \geq-\widetilde{\varepsilon} .
$$

Relations (2.2) and (2.3), the definitions of $\widetilde{\varphi}_{i, k}$, and the maximum principle for analytic functions imply that

$$
\ln \left|\frac{\widetilde{\varphi}_{i(k), k}(z)}{\widetilde{\varphi}_{i(k), k}\left(z_{k}\right)}\right| \leq 2 \widetilde{\varepsilon}\left|z_{k}\right|^{\rho}, \quad z \in B\left(z_{k}, 6 \delta\left|z_{k}\right|\right)
$$

Applying a lower estimate for the absolute value of an analytic function (see [2]), we see that in the above disk, but outside some exceptional small disks with total sum of the radii equal to $(\widetilde{r}-r) \delta\left|z_{k}\right| / 2,0<r<\widetilde{r}$, we have

$$
\ln \left|\frac{\widetilde{\varphi}_{i(k), k}(z)}{\widetilde{\varphi}_{i(k), k}\left(z_{k}\right)}\right| \geq-2 a \widetilde{\varepsilon}\left|z_{k}\right|^{\rho}, \quad k \geq k_{2},
$$

where $a=2+\ln (3 e / 2 \eta), \eta=(\widetilde{r}-r) / 8$. Moreover, the proof of the Cartan lemma on the lower estimate for a polynomial (see, e.g., [2]) shows that each of the exceptional disks contains at least one zero of the function $\widetilde{\varphi}_{i(k), k}$. By construction, our assumptions imply that $\widetilde{\varphi}_{i(k), k}$ has no zeros in the disk $B\left(z_{k}, \widetilde{r} \delta\left|z_{k}\right|\right)$. Therefore, the disk $B\left(z_{k}, r \delta\left|z_{k}\right|\right)$ does not intersect the exceptional disks. Using (2.4) and (2.6), the fact that $h_{\psi_{i(k)}}$ is homogeneous, the definition of the functions $\widetilde{\varphi}_{i(k), k}$, and inequality (2.7), we obtain

$$
\begin{array}{r}
\max _{1 \leq i \leq n}\left[\ln \left|\frac{\psi_{i}(z)}{q_{\delta}\left(z, z_{k}, \Lambda\right)}\right|-h_{\psi_{i}}(z)\right] \geq \ln \left|\widetilde{\varphi}_{i(k)}(z)\right|-h_{\psi_{i(k)}}(z) \\
\geq-(1-\delta)^{-\rho}(2+2 a) \widetilde{\varepsilon}|z|^{\rho}, \quad z \in B\left(z_{k}, r \delta\left|z_{k}\right|\right), \quad k \geq k_{2} .
\end{array}
$$

Now, suppose that at least one of the functions $\psi_{1}, \ldots, \psi_{n}$ has noncommon zeros in the disk $B\left(z_{k}, \widetilde{r} \delta\left|z_{k}\right|\right)$. Then, by the second condition of Definition 2 and the definition of the $\varphi_{i, k}$, for any index $1 \leq p \leq n$ and all $\lambda_{l}^{p} \in B\left(z_{k}, \widetilde{r} \delta\left|z_{k}\right|\right)$ there exists $i(p, l) \in\{1, \ldots, n\}$ such that the following inequalities are fulfilled:

$$
\begin{aligned}
\ln \left|\varphi_{i(p, l), k}\left(\lambda_{l}^{p}\right)\right|+\ln \left|\widetilde{q}_{\tilde{r} \delta}\left(\lambda_{l}^{p}, z_{k}, \Lambda_{i(p, l)}\right)\right|-h_{\psi_{i(p, l)}}\left(\lambda_{l}^{p}\right) & \geq-\widetilde{\varepsilon}\left|\lambda_{l}^{p}\right|^{\rho}, \\
\ln \left|\widetilde{\varphi}_{i(p, l), k}\left(\lambda_{l}^{p}\right)\right|-h_{\psi_{i(p, l)}}\left(\lambda_{l}^{p}\right) & \geq-\widetilde{\varepsilon}\left|\lambda_{l}^{p}\right|^{\rho}, \quad k \geq k_{2} .
\end{aligned}
$$

We denote by $\Gamma(k)$ the set of all such indices $i(p, l)$ for $p=1, \ldots, n$ and for $l$ such that $\lambda_{l}^{p} \in B\left(z_{k}, \widetilde{r} \delta\left|z_{k}\right|\right)$. The above inequalities show that for each $i \in \Gamma(k), k \geq k_{1}$, there exists a pair of indices $p(i), l(i)$ with

$$
\begin{aligned}
& \ln \left|\varphi_{i, k}\left(\lambda_{l(i)}^{p(i)}\right)\right|-h_{\psi_{i}}\left(\lambda_{l(i)}^{p(i)}\right) \geq-\widetilde{\varepsilon}\left|\lambda_{l(i)}^{p(i)}\right|^{\rho}, \quad \lambda_{l(i)}^{p(i)} \in B\left(z_{k}, \widetilde{r} \delta\left|z_{k}\right|\right) . \\
& \ln \left|\widetilde{\varphi}_{i, k}\left(\lambda_{l(i)}^{p(i)}\right)\right|-h_{\psi_{i}}\left(\lambda_{l(i)}^{p(i)}\right) \geq-\widetilde{\varepsilon}\left|\lambda_{l(i)}^{p(i)}\right|^{\rho},
\end{aligned}
$$

Moreover,

$$
\begin{array}{r}
\max _{i \in \Gamma(k)} \ln \left|\widetilde{q}_{\widetilde{r} \delta}\left(\lambda_{l}^{p}, z_{k}, \Lambda_{i}\right)\right| \geq-3 \widetilde{\varepsilon}(1+\delta)^{\rho}\left|z_{k}\right|^{\rho}, \\
1 \leq p \leq n, \quad \lambda_{l}^{p} \in B\left(z_{k}, \widetilde{r} \delta\left|z_{k}\right|\right), \quad k \geq k_{2} .
\end{array}
$$


Applying the inequalities (2.9), estimates (2.2), (2.3), and (2.4), the definition of the functions $\varphi_{i, k}$ and $\widetilde{\varphi}_{i, k}$, and the maximum principle, we see that, for each $k \geq k_{2}$ and all $z \in B\left(\lambda_{l(i)}^{p(i)}, 12 \delta\left|\lambda_{l(i)}^{p(i)}\right|\right)$ we have

$$
\begin{aligned}
& \ln \left|\frac{\varphi_{i, k}(z)}{\varphi_{i, k}\left(\lambda_{l(i)}^{p(i)}\right)}\right| \leq 3 \widetilde{\varepsilon}\left|\lambda_{l(i)}^{p(i)}\right|^{\rho} \leq 3 \widetilde{\varepsilon}(1+\delta)^{\rho}\left|z_{k}\right|^{\rho}, \\
& \ln \left|\frac{\widetilde{\varphi}_{i, k}(z)}{\widetilde{\varphi}_{i, k}\left(\lambda_{l(i)}^{p(i)}\right)}\right| \leq 3 \widetilde{\varepsilon}\left|\lambda_{l(i)}^{p(i)}\right|^{\rho} \leq 3 \widetilde{\varepsilon}(1+\delta)^{\rho}\left|z_{k}\right|^{\rho},
\end{aligned}
$$

Arguing as in the proof of (2.7), from (2.11) we obtain the inequality

$$
\ln \left|\frac{\varphi_{i, k}(z)}{\varphi_{i, k}\left(\lambda_{l(i)}^{p(i)}\right)}\right| \geq-3 a \widetilde{\varepsilon}(1+\delta)^{\rho}\left|z_{k}\right|^{\rho}, \quad i \in \Gamma(k), \quad z \in B\left(z_{k}, r \delta\left|z_{k}\right|\right),
$$

where $a=2+\ln (3 e / 2 \eta), \eta=(\widetilde{r}-r) / 8$, and $r \in(0, \widetilde{r})$.

Let $m_{i, k}$ denote the number of noncommon zeros of $\psi_{i}$ in the disk $B\left(z_{k}, \delta\left|z_{k}\right|\right)$. By construction, these points are also zeros of $\widetilde{\varphi}_{i, k}$. Since the funcntion $\widetilde{\varphi}_{i, k}$ is entire, by 10, Lemma 4.1] and (2.11) we have the estimate

$$
m_{i, k} \leq 3 \widetilde{\varepsilon}(1+\delta)^{\rho}\left|z_{k}\right|^{\rho}, \quad i \in \Gamma(k), \quad k \geq k_{2} .
$$

By (2.10) and Theorem 2.1, the inequality

$$
\max _{i \in \Gamma(k)} \ln \left|\widetilde{q}_{\widetilde{r} \delta}\left(z, z_{k}, \Lambda_{i(p, l)}\right)\right| \geq-8 \widetilde{\varepsilon}(1+\delta)^{\rho}\left|z_{k}\right|^{\rho}, \quad k \geq k_{2},
$$

is fulfilled in each disk $B\left(z_{k}, \tilde{r} \delta\left|z_{k}\right|\right)$. Combining this with (2.4), (2.9)), and (2.12), we obtain

$$
\begin{aligned}
\max _{1 \leq i \leq n} & {\left[\ln \left|\frac{\psi_{i}(z)}{q_{\delta}\left(z, z_{k}, \Lambda\right)}\right|-h_{\psi_{i}}(z)\right] } \\
& \geq \max _{i \in \Gamma(k)}\left[\ln \left|\varphi_{i, k}(z)\right|+\ln \left|\widetilde{q}_{\tilde{r} \delta}\left(z, z_{k}, \Lambda_{i}\right)\right|-h_{\psi_{i}}(z)\right] \\
& \geq-\frac{2+(3 a+5)(1+\delta)^{\rho}}{(1-\delta)^{\rho}} \widetilde{\varepsilon}|z|^{\rho}, \quad z \in B\left(z_{k}, r \delta\left|z_{k}\right|\right), \quad k \geq k_{2} .
\end{aligned}
$$

Consider the disk $B\left(z_{k}, \tilde{r} \delta\left|z_{k}\right|\right)$ with $k \geq k_{2}$. If none of the functions $\varphi_{1}, \ldots, \varphi_{n}$ has a noncommon zero in that disk, then we have (2.8). Otherwise, estimate (2.13) is valid. We put $\varepsilon=\left(2+(3 a+5)(1+\delta)^{\rho}\right) /(1-\delta)^{\rho}$ and $k_{0}=k_{2}$, and choose $\delta(r, \varepsilon)>0$ sufficiently small; this yields the inequality required in Definition 1 .

We present a general example of a system of two functions having regular growth along a ray $\{t y, t>0\}$. For this, we need the following definition, which is equivalent to the classical definition of regular growth in the sense of Levin-Pflüger.

Definition 3 ([8, 10]). A function $\psi(z)$ is said to have regular growth along a ray $t y, y \in \mathbf{S}$, if there exists a sequence $\left\{z_{k}\right\} \in E(y)$ such that

$$
\lim _{k \rightarrow \infty} \ln \left|\psi\left(z_{k}\right)\right| /\left|z_{k}\right|^{\rho}=h_{\psi}(y) .
$$

Let $\psi_{1}$ and $\psi_{2}$ be entire functions of order (at most) $\rho$ and of finite type (with order $\rho$ ). Suppose that each of them (separately) has regular growth along a ray $\{t y, t>0\}, y \in \mathbf{S}$. Then, by Definition 3 , there is a sequence $\left\{z_{k}\right\} \in E(y)$ (one and the same for $\psi_{1}$ and $\psi_{2}$ ) such that

$$
\lim _{k \rightarrow \infty} \ln \left|\psi_{i}\left(z_{k}\right)\right| /\left|z_{k}\right|^{\rho}=h_{\psi_{i}}(y), \quad i=1,2
$$


Next, the noncommon zeros $\Lambda_{1}=\left\{\lambda_{j}^{1}\right\}$ and $\Lambda_{2}=\left\{\lambda_{j}^{2}\right\}$ of $\psi_{1}$ and $\psi_{2}$ satisfy the estimate

$$
\left|\lambda_{k+1}^{i}\right|-\left|\lambda_{k}^{i}\right|>\frac{1}{\varepsilon}\left|\lambda_{k}^{i}\right|^{1-\rho}, \quad i=1,2, \quad k=1,2, \ldots,
$$

where $\varepsilon>0$. We show that if

$$
\begin{array}{ll}
\min _{j}\left|\lambda_{k}^{1}-\lambda_{j}^{2}\right| /\left|\lambda_{k}^{1}\right|>\exp \left[-\varepsilon\left|\lambda_{k}^{1}\right|^{\rho}\right], & k=1,2, \ldots, \\
\min _{k}\left|\lambda_{j}^{2}-\lambda_{k}^{1}\right| /\left|\lambda_{j}^{2}\right|>\exp \left[-\varepsilon\left|\lambda_{j}^{2}\right|^{\rho}\right], & j=1,2, \ldots,
\end{array}
$$

then the system $\left(\psi_{1}, \psi_{2}\right)$ has regular growth along the ray $\{t y, t>0\}$.

Obviously, we can find $R, \delta^{\prime}>0$ such that

$$
\ln \left|\psi_{i}(t z)\right| / t^{\rho} \leq h_{\psi_{i}}(y)+\varepsilon, \quad z \in B\left(y, 6 \delta^{\prime}\right), \quad t>R, \quad i=1,2 .
$$

Since the functions $h_{\psi_{i}}(z)$ are continuous, we may assume that

$$
\left|h_{\psi_{i}}(y)-h_{\psi_{i}}(z)\right|<\varepsilon, \quad z \in B\left(y, \delta^{\prime}\right) .
$$

By assumption, each of the functions $\psi_{1}$ and $\psi_{2}$ has regular growth along $\{t y, t>0\}$. Therefore, for some index $k_{1}$ such that $\left|z_{k_{1}}\right|>R$ we have

$$
\ln \left|\psi_{i}\left(z_{k}\right)\right| /\left|z_{k}\right|^{\rho}-h_{\psi_{i}}(y) \geq-\varepsilon, \quad i=1,2,
$$

for $k \geq k_{1}$.

For each $k \geq k_{1}$ we consider the functions

$$
\varphi_{i, k}(z)=\psi_{i}(z)\left[q_{\delta}\left(z, z_{k}, \Lambda_{i}\right) q_{\delta}\left(z, z_{k}, \Lambda\right)\right]^{-1}, \quad \delta \in\left(0, \delta^{\prime}\right) .
$$

Arguing as in the proof of (2.7), we obtain

$$
\ln \left|\frac{\varphi_{i, k}(z)}{\varphi_{i, k}\left(z_{k}\right)}\right| \geq-2 a \varepsilon\left|z_{k}\right|^{\rho}, \quad z \in B\left(z_{k}, r \delta\left|z_{k}\right|\right), \quad k \geq k_{1},
$$

where $r \in(0,1), a=2+\ln (3 e / 2 \eta)$, and $\eta=(1-r) / 8$.

Consider the disk $B\left(z_{k}, r \delta\left|z_{k}\right|\right)$. If for some $i=1,2$ the set $\Lambda_{i} \cap B\left(z_{k}, r \delta\left|z_{k}\right|\right)$ is empty, then all conditions of Definition 2 are satisfied for that disk. (The first condition is fulfilled automatically by the regularity of the growth of each of the functions $\psi_{1}$ and $\psi_{2}$, and the second is implied by estimates (2.14) and (2.16).) To simplify the notation, we put $\Lambda_{i} \cap B\left(z_{k}, r \delta\left|z_{k}\right|\right)=\left\{\lambda_{1}^{i}, \ldots, \lambda_{m_{i}}^{i}\right\}, i=1,2$. For each $l=1, \ldots, m_{1}$, there exists an index $j$ such that $\left|\lambda_{j+1}^{2}\right|>\left|\lambda_{l}^{1}\right|>\left|\lambda_{j}^{2}\right|$ (the cases where $\left|\lambda_{l}^{1}\right|>\left|\lambda_{m_{2}}^{2}\right|$ and $\left|\lambda_{l}^{1}\right|<\left|\lambda_{1}^{2}\right|$ are analyzed similarly). Suppose $\rho \geq 1$. Then

$$
\left|\lambda_{k+1}^{i}\right|-\left|\lambda_{k}^{i}\right|>1 /\left(\varepsilon\left|\lambda_{m_{i}}^{i}\right|^{\rho-1}\right), \quad i=1,2,
$$

whence

$$
\begin{aligned}
& \left|\lambda_{l}^{1}-\lambda_{k}^{2}\right| \geq\left|\lambda_{l}^{1}\right|-\left|\lambda_{k}^{2}\right|>\left|\lambda_{j}^{2}\right|-\left|\lambda_{k}^{2}\right|>\frac{(j-k)}{\varepsilon\left|\lambda_{m_{2}}^{2}\right|^{\rho-1}}, \quad k=1, \ldots, j-1 ; \\
& \left|\lambda_{l}^{1}-\lambda_{k}^{2}\right| \geq\left|\lambda_{k}^{2}\right|-\left|\lambda_{l}^{1}\right|>\left|\lambda_{k}^{2}\right|-\left|\lambda_{j+1}^{2}\right|>\frac{(k-j-1)}{\varepsilon\left|\lambda_{m_{2}}^{2}\right|^{\rho-1}}, \quad k=j+2, \ldots, m_{2} ; \\
& \frac{\left|\lambda_{l}^{1}-\lambda_{k}^{2}\right|}{\left|\lambda_{l}^{1}\right|}>\exp \left[-\varepsilon\left|\lambda_{l}^{1}\right|^{\rho}\right], \quad k=j, j+1 .
\end{aligned}
$$

The Taylor expansion of the exponential function implies the following inequality:

$$
\exp (t)>\frac{t^{n}}{n !}, \quad n=1,2, \ldots, t>0 .
$$

In particular,

$$
\exp \left(c|z|^{\rho}\right)>\frac{\left(c|z|^{\rho}\right)^{n}}{n !}, \quad n=1,2, \ldots
$$


where $c$ is some positive constant. Applying (2.17) and the latter inequality, we see that the following estimates are true for some $k_{2} \geq k_{1}$ :

$$
\begin{aligned}
& \left|q_{\delta}\left(\lambda_{l}^{1}, z_{k}, \Lambda_{2}\right)\right|=\prod_{k=1}^{m_{2}} \frac{\left|\lambda_{l}^{1}-\lambda_{k}^{2}\right|}{\left|z_{k}-\lambda_{k}^{2}\right|}>\prod_{k=1}^{m_{2}} \frac{\left|\lambda_{l}^{1}-\lambda_{k}^{2}\right|}{2 \delta\left|z_{k}\right|}>\prod_{k=j}^{j+1} \frac{\left|\lambda_{l}^{1}-\lambda_{k}^{2}\right|}{\widetilde{\delta}\left|\lambda_{l}^{1}\right|} \prod_{k=1, k \neq j, j+1}^{m_{2}} \frac{\left|\lambda_{l}^{1}-\lambda_{k}^{2}\right|}{\widetilde{\delta}\left|\lambda_{m_{2}}^{2}\right|} \\
& >\frac{1}{\widetilde{\delta}^{2}} \exp \left[-2 \varepsilon\left|\lambda_{l}^{1}\right|^{\rho}\right] \frac{(j-1) !}{\left(\widetilde{\delta} \varepsilon\left|\lambda_{m_{2}}^{2}\right| \rho\right)^{j-1}} \frac{\left(m_{2}-j-1\right) !}{\left(\widetilde{\delta} \varepsilon\left|\lambda_{m_{2}}^{2}\right| \rho\right)^{m_{2}-j-1}} \\
& >\exp \left[-C \varepsilon\left|\lambda_{l}^{1}\right|^{\rho}\right], \quad l=1, \ldots, m_{1}, \quad k \geq k_{2},
\end{aligned}
$$

where $\widetilde{\delta}=2 \delta /(1-\delta)$ and $C$ is a positive constant. In a similar way, we obtain

$$
\left|q_{\delta}\left(\lambda_{l}^{2}, z_{k}, \Lambda_{1}\right)\right|>\exp \left[-C \varepsilon\left|\lambda_{l}^{2}\right|^{\rho}\right], \quad l=1, \ldots, m_{2}, k \geq k_{2} .
$$

Consequently, for $p=1,2$ we have

$$
\max _{i=1,2}\left|q_{\delta}\left(\lambda_{l}^{p}, z_{k}, \Lambda_{i}\right)\right|>\exp \left[-C \varepsilon\left|\lambda_{l}^{p}\right|^{\rho}\right], \quad l=1, \ldots, m_{p}, \quad k \geq k_{2} .
$$

Combining this with estimates (2.14), (2.15), and (2.16), we see that for $p=1,2$ and each $l=1, \ldots, m_{p}$ the inequality

$$
\begin{aligned}
\max _{i=1,2} & {\left[\ln \left|\frac{\psi_{i}\left(\lambda_{l}^{p}\right)}{q_{\delta}\left(\lambda_{l}^{p}, z_{k}, \Lambda\right)}\right|-h_{\psi_{i}}\left(\lambda_{l}^{p}\right)\right] } \\
& =\max _{i=1,2}\left[\ln \left|\varphi_{i, k}\left(\lambda_{l}^{p}\right)\right|+\ln \left|q_{\delta}\left(\lambda_{l}^{p}, z_{k}, \Lambda_{i}\right)\right|-h_{\psi_{i}}\left(\lambda_{l}^{p}\right)\right]>-C_{1} \varepsilon\left|\lambda_{l}^{p}\right|^{\rho}
\end{aligned}
$$

is valid for $k \geq k_{2}$, where $C_{1}>0$. Putting $k_{0}=k_{2}$, we take $C_{1} \varepsilon$ in the role of $\varepsilon$ in Definition 2 Thus, all requirements of Definition 2 are fulfilled, which means that the system $\left(\psi_{1}, \psi_{2}\right)$ has regular growth along the ray $\{t y, t>0\}$ provided $\rho \geq 1$. The case of $\rho<1$ is treated similarly, with the use of the inequalities

$$
\left|\lambda_{k+1}^{i}\right|-\left|\lambda_{k}^{i}\right|>1 /\left(\varepsilon\left|\lambda_{1}^{i}\right|^{\rho-1}\right), \quad\left|\lambda_{1}^{i}\right|>1, \quad i=1,2 .
$$

We note that the above example can be extended to the case of a system of $n$ functions, with only minor changes in the proof.

Now we pass to the notion of weak regular growth for a system of functions; this notion was also introduced in [5].

Definition $4([5])$. We say that a system $\left(\psi_{1}, \ldots, \psi_{n}\right)$ of entire functions of order (at most) $\rho$ and of finite type (with order $\rho$ ) has weak regular growth along a ray $t y(y \in S)$ if for any $r>\tau>1$ there exists $\delta_{0}>0$ with the following property: for every $\delta \in\left(0, \delta_{0}\right]$ we can find a sequence $\left\{z_{k}\right\} \in E(y, \delta)$ and a number $A>0$ such that

$$
\max _{1 \leq i \leq n} \ln \left|\psi_{i}(z) / q_{r \delta}\left(z, z_{k}, \Lambda\right)\right| \geq-A|z|^{\rho}, \quad z \in B\left(z_{k}, \tau \delta\left|z_{k}\right|\right), \quad k \geq 1,
$$

where $\Lambda=\left\{\lambda_{j}\right\}_{j=1}^{\infty}$ is the set of noncommon zeros of the functions $\psi_{i}$ (with regard to multiplicity).

Like for the notion of regular growth, we give another definition of weak regular growth for a system of functions and prove the equivalence of two definitions.

Definition 5. A system $\left(\psi_{1}, \ldots, \psi_{n}\right)$ of entire functions of order (at most) $\rho$ and of finite type (with order $\rho$ ) in $\mathbb{C}$ is said to have weak regular growth along a ray ty $(y \in S)$ if there exists a number $\delta_{0}>0$ with the following property: for any $\delta \in\left(0, \delta_{0}\right]$ there are numbers $r_{0}$ and $A>0$ such that for all $z_{0} \in\{t y, t>0\}, z_{0} \notin \bigcup_{i=1}^{n} \Lambda_{i},\left|z_{0}\right| \geq r_{0}$, all indices $1 \leq p \leq n$, and all $\lambda_{l}^{p} \in \Lambda_{p} \cap B\left(z_{0}, \delta\left|z_{0}\right|\right)$ we have

$$
\max _{1 \leq i \leq n} \ln \left|q_{\delta}\left(\lambda_{l}^{p}, z_{0}, \Lambda_{i}\right)\right| \geq-A\left|z_{0}\right|^{\rho},
$$


where $\Lambda_{i}=\left\{\lambda_{j}^{i}\right\}_{j=1}^{\infty}$ is the set of noncommon zeros of the function $\psi_{i}$ (with regard to multiplicity).

Proposition 2. Definitions 4 and 5 are equivalent.

Proof. Suppose the conditions listed in Definition 4 are fulfilled with $A$ and $\delta_{0}$ replaced by $\widetilde{A}$ and $\widetilde{\delta}_{0}$, respectively. Since the functions $\psi_{1}, \ldots, \psi_{n}$ are of order (at most) $\rho$ and of finite type (with order $\rho$ ), we have

$$
\ln \left|\psi_{i}(z)\right| \leq a|z|^{\rho}, \quad|z|>R
$$

for any $1 \leq i \leq n$, where $a, R>0$. We choose $k_{1}$ so as to ensure that for all $k \geq k_{1}$ the disk $B\left(z_{k}, 12 \delta\left|z_{k}\right|\right)$ be disjoint from $B(0, R)$, and put

$$
\varphi_{i, k}(z)=\psi_{i}(z)\left[\widetilde{q}_{4 \delta}\left(z, z_{k}, \Lambda_{i}\right) q_{5 \delta}\left(z, z_{k}, \Lambda\right)\right]^{-1}, \quad \delta \in\left(0, \widetilde{\delta}_{0} / 4\right]
$$

Relations (2.2) and (2.18), the inequality in Definition 4 and the maximum principle imply the estimate

$$
\max _{1 \leq i \leq n} \ln \left|\widetilde{q}_{4 \delta}\left(z, z_{k}, \Lambda_{i}\right)\right| \geq-A^{\prime}|z|^{\rho}, \quad z \in B\left(z_{k}, 4 \delta\left|z_{k}\right|\right), \quad k \geq k_{1},
$$

where $A^{\prime}=\widetilde{A}+a(1+10 \delta)^{\rho}(1-4 \delta)^{-\rho}$. Let $z_{0}$ be a point of the ray $\{t y, t>0\}$ such that $\left|z_{0}\right|>\left|z_{k_{1}+1}\right|$. Obviously, there exists an index $k>k_{1}$ such that $\left|z_{k-1}\right|<\left|z_{0}\right| \leq\left|z_{k}\right|$ and the disk $B\left(z_{k}, 4 \delta\left|z_{k}\right|\right)$ contains $B\left(z_{0}, \delta\left|z_{0}\right|\right)$. By using the above inequality and (2.2), now it is easy to obtain the estimate

$$
\begin{array}{r}
\max _{1 \leq i \leq n} \ln \left|q_{\delta}\left(z, z_{0}, \Lambda_{i}\right)\right| \geq \max _{1 \leq i \leq n} \ln \left|\widetilde{q}_{4 \delta}\left(z, z_{k}, \Lambda_{i}\right)\right| \geq-A^{\prime}|z|^{\rho}, \\
z \in B\left(z_{k}, \delta\left|z_{k}\right|\right), \quad k \geq k_{1} .
\end{array}
$$

Putting $A=A^{\prime}, \delta_{0}=\widetilde{\delta}_{0} / 4, r_{0}=\left|z_{k_{1}+1}\right|$, we see that the inequalities in Definition 5 are fulfilled automatically.

Now, suppose that the requirements of Definition 5 are fulfilled with $A$ and $\delta_{0}$ replaced by $\widetilde{A}$ and $\widetilde{\delta}$, respectively. Let $r>\tau>1$, and let $\delta \in\left(0, \widetilde{\delta}_{0} / r\right]$. The proof of the theorem about the lower estimate for an entire function of finite type on a system of circles (see, e.g., [2]) shows that there exists a sequence $\left\{z_{k}\right\} \in E(y, \delta)$ and a number $C>0$ such that

$$
\ln \left|\psi_{i}\left(z_{k}\right)\right| \geq-C\left|z_{k}\right|^{\rho}, \quad k \geq 1, \quad i=1, \ldots, n .
$$

We choose an index $k_{1}$ so that $(1-6 \delta)\left|z_{k_{1}}\right|>\max \left\{r_{0}, R\right\}$, where $r_{0}$ is as in Definition 5 and put

$$
\varphi_{i, k}(z)=\psi_{i}(z)\left[\psi_{i}\left(z_{k}\right) q_{r \delta}\left(z, z_{k}, \Lambda_{i}\right) q_{r \delta}\left(z, z_{k}, \Lambda\right)\right]^{-1} .
$$

It is easily seen that $\varphi_{i, k}\left(z_{k}\right)=1$. From (2.2), (2.18), and (2.19) we obtain

$$
\ln \left|\varphi_{i, k}(z)\right| \leq a^{\prime}\left|z_{k}\right|^{\rho}, \quad z \in B\left(z_{k}, 6 r \delta\left|z_{k}\right|\right), \quad k \geq k_{1},
$$

where $a^{\prime}=(1+6 r \delta)^{\rho} a+C$. The theorem on the lower estimate for analytic functions implies that in the disk $B\left(z_{k}, r \delta\left|z_{k}\right|\right)$, but outside some exceptional disks with the sum of radii $(r-\tau) \delta\left|z_{k}\right|$, we have

$$
\ln \left|\varphi_{i, k}(z)\right| \geq-H(\eta) a^{\prime}\left|z_{k}\right|^{\rho}, \quad k \geq k_{1}
$$

where $\eta=(r-\tau) /(8 r), H(\eta)=2-\ln (3 e /(2 \eta))$. Since $\varphi_{i, k}$ has no zeros in $B\left(z_{k}, r \delta\left|z_{k}\right|\right)$, the inequality obtained above is valid everywhere in the disk $B\left(z_{k}, \tau \delta\left|z_{k}\right|\right)$.

Let $m_{i, k}$ denote the number of zeros of $\varphi_{i}$ in $B\left(z_{k}, r \delta\left|z_{k}\right|\right)$. From [10, Lemma 4.1] and (2.18), (2.19), it follows that

$$
m_{i, k} \leq \max _{z \in \partial B\left(z_{k}, 3 r \delta\left|z_{k}\right|\right)} \ln \left|\frac{\psi_{i}(z)}{\psi_{i}\left(z_{k}\right)}\right| \leq a(1+3 r \delta)^{\rho}\left|z_{k}\right|^{\rho}, \quad k \geq k_{1} .
$$


The conditions of Definition 5 imply that for any $1 \leq p \leq n$ and all $\lambda_{l}^{p} \in \Lambda_{p} \cap B\left(z_{k}, \delta\left|z_{k}\right|\right)$ we have

$$
\max _{1 \leq i \leq n} \ln \left|q_{r \delta}\left(\lambda_{l}^{p}, z_{k}, \Lambda_{i}\right)\right| \geq-\widetilde{A}\left|z_{k}\right|^{\rho}, \quad k \geq k_{1} .
$$

Now we apply Theorem 2.1 The above two inequalities imply that if $k \geq k_{1}$, then for all $z \in B\left(z_{k}, r \delta\left|z_{k}\right|\right)$ we have

$$
\max _{1 \leq i \leq n} \ln \left|q_{r \delta}\left(z, z_{k}, \Lambda_{i}\right)\right| \geq-\left(\widetilde{A}+a(1+3 r \delta)^{\rho}\right)\left|z_{k}\right|^{\rho} .
$$

Everything is ready for the proof of the inequality in Definition 4 We use the definition of the functions $\varphi_{i, k}$, the inequality above, and (2.19), (2.20) to deduce the estimate

$$
\max _{1 \leq i \leq n} \ln \left|\psi_{i}(z) / q_{r \delta}\left(z, z_{k}, \Lambda\right)\right| \geq-A_{1}|z|^{\rho}, \quad z \in B\left(z_{k}, \tau \delta\left|z_{k}\right|\right), \quad k \geq k_{1},
$$

where $A_{1}=\left(\widetilde{A}+a(1+3 r \delta)^{\rho}+H(\eta) a^{\prime}\right)(1-\tau \delta)^{-\rho}$. So, the inequality in question is fulfilled with $A_{1}, \widetilde{\delta}_{0} / r$, and $k_{1}$ in place of $A_{0}, \delta_{0}$, and $k_{0}$, respectively.

\section{§3. Solvability of Systems of NONhOMOGENEOUS CONVOLUTION EQUATIONS}

In this section we prove the sufficient conditions of solvability. Our approach differs substantially from that presented in [5]. All lemmas formulated without proof can be found in [5].

For a convex domain $D \subset \mathbb{C}$ we put

$$
I_{D}=\left\{z \in \mathbb{C}: H_{D}(z)=+\infty\right\} .
$$

If $D$ is bounded, then, obviously, $I_{D}=\varnothing$.

Lemma 3.1. Let $D$ be a convex domain in $\mathbb{C}$, let $K$ be a compact convex subset of $D$, and let $a$ be a positive number. Suppose that

$$
H_{K}(z)+a|z|<H_{D}(z), \quad z \in \mathbb{C} \backslash\{0\} .
$$

Moreover, suppose that $X \subset S$ is a compact subset of the interior int $I_{D}$ of the set $I_{D}$. Then for any $A>0$ there exists a compact set $L \subset D$ such that $K \subset L$ and the following inequalities are fulfilled:

$$
H_{L}(z)+a|z|<H_{D}(z), \quad z \in \mathbb{C} \backslash\{0\} ; \quad H_{L}(z)>A|z|, \quad z /|z| \in X .
$$

We fix an arbitrary $\varepsilon>0$ and a closed subset $Z$ of the unit circle $\mathbf{S}$. Let $\left(f_{1}, \ldots, f_{n}\right)$ be a system of entire functions of exponential type in $\mathbb{C}$ and satisfying the following conditions:

i) $\left(f_{1}, \ldots, f_{n}\right)$ has regular growth on $Z$ (i.e., on every ray $t y, y \in Z$ );

ii) $\left(f_{1}, \ldots, f_{n}\right)$ has weak regular growth on $\mathbf{S} \backslash Z$.

Since the indicators $h_{f_{i}}(z)$ are uniformly continuous on compact subsets of $\mathbb{C}$, there is a number $\delta^{\prime}>0$ such that $32 \delta^{\prime}<1$ and

$$
\sum_{i=1}^{n}\left|h_{f_{i}}(z)-h_{f_{i}}(y)\right|<\varepsilon, \quad y \in B\left(z, 16 \delta^{\prime}\right), \quad z \in \mathbf{S} .
$$

Since $h_{f_{i}}$ is the indicator diagram for $f_{i}$, we have

$$
\ln \left|f_{i}(z)\right| \leq h_{f_{i}}(z)+\varepsilon|z|+C^{\prime}, \quad z \in \mathbb{C}, \quad i=1, \ldots, n
$$

(see [1]), where $C^{\prime}>0$ is some constant depending on $\varepsilon>0$.

Now, let $y \in Z$. By i), the system $\left(f_{1}, \ldots, f_{n}\right)$ has regular growth along the ray $t y$. For a fixed $\varepsilon>0$ and $r=1 / 2$, we denote by $\delta^{\prime}(y)$ the quantity $\delta(r, \varepsilon) / 4$, where $\delta(r, \varepsilon)$ is the number occurring in Definition 1. For each $y \in Z$ we fix $\delta(y)>0$ so that

$$
\delta(y) \leq \min \left\{\delta^{\prime}, \delta^{\prime}(y)\right\} .
$$


The disks $B(y, \delta(y) / 4), y \in Z$, cover $Z$; we extract a finite subcover $B\left(y_{l}, \delta_{l} / 4\right)$ (here $\left.\delta_{l}=\delta\left(y_{l}\right)\right)$. Let $\left\{z_{k, l}\right\}_{k=1}^{\infty}$ be a sequence $\left\{z_{k}\right\}_{k=k_{0}}^{\infty} \in E\left(y_{l}\right)$ satisfying the inequalities in Definition 11 In accordance with the definition of the classes $E\left(y_{l}\right)$ and $E\left(y_{l}, \delta\right)$, by deleting finitely many elements from the sequence $\left\{z_{k, l}\right\}$ we can arrange that $\left\{z_{k, l}\right\} \in$ $E\left(y_{l}, \delta_{l} / 2\right)$. Now, it is easily seen that for each $l=1, \ldots, l_{0}$ we can find a number $R_{l}>0$ such that, off the disk $B\left(0, R_{l}\right)$, the angle with vertex at the origin and generated by the disk $B\left(y_{l}, \delta_{l} / 4\right)$ is covered by the union $\bigcup_{k} B\left(z_{k, l}, \delta\left|z_{k, l}\right|\right)$. We denote

$$
\Sigma=\mathbf{S} \backslash\left[\bigcup_{i=1}^{l_{0}} B\left(y_{l}, \delta_{l} / 4\right)\right] .
$$

Then there is a number $\widetilde{\delta}>0$ such that $16 \widetilde{\delta}<1$ and for each $x \in \Sigma$ the disk $B(x, 8 \widetilde{\delta})$ is compactly contained in the angle with vertex at the origin and generated by the set $\mathbf{S} \backslash Z$. Since the functions $f_{i}(z), i=1, \ldots, n$, are of exponential type, for some $A^{\prime}, B^{\prime}>0$ we have

$$
\ln \left|f_{i}(z)\right| \leq A^{\prime}|z|+B^{\prime}, \quad z \in \mathbb{C}, \quad i=1, \ldots, n .
$$

By condition ii), for $\tau=2, r=4$, and every $x \in \Sigma$, we can find a number $\delta_{0}=\delta_{0}(x)$ as in Definition 4. For every $x \in \Sigma$ we fix an arbitrary number $\widetilde{\delta}(x)>0$ satisfying

$$
\widetilde{\delta}(x) \leq \min \left\{\delta^{\prime}, \widetilde{\delta}, \delta_{0}(x)\right\} .
$$

As before, we find points $x_{l} \in \Sigma, l=1, \ldots, l_{1}$, such that

$$
\Sigma \subset \bigcup_{l=1}^{l_{1}} B\left(x_{l}, \widetilde{\delta}_{l} / 2\right)
$$

where $\widetilde{\delta}_{l}=\widetilde{\delta}\left(x_{l}\right)$. By Definition 4 for each $l=1, \ldots, l_{1}$ we can choose a sequence $\left\{w_{k, l}\right\} \in E\left(x_{l}, \widetilde{\delta}_{l}\right)$ and a number $A_{l}>0$ so that the inequality in Definition 4 be fulfilled for $\psi_{i}=f_{i}, i=1, \ldots, n$, with $\rho=1$ and with $\left\{w_{k, l}\right\}, A_{l}$, and $\widetilde{\delta}_{l}$ in the role of $\left\{z_{k}\right\}, A$, and $\delta$, respectively. Again as before, there are numbers $T_{l}>0, l=1, \ldots, l_{1}$, such that off the disk $B\left(0, T_{l}\right)$ the angle spanned by the disk $B\left(x_{l}, \widetilde{\delta}_{l} / 2\right)$ is covered by the union $\bigcup_{k=1}^{\infty} B\left(w_{k, l}, 2 \widetilde{\delta}_{l}\left|w_{k, l}\right|\right)$.

We put $\Theta=\max _{1 \leq l \leq l_{1}} A_{l}$ and denote by $U_{k, l}^{i}$ (respectively, $\widetilde{U}_{k, l}^{i}$ ) the open set of points $z \in B\left(z_{k, l}, 2 \delta_{l}\left|z_{k, l}\right|\right)$ (respectively, $\left.z \in B\left(w_{k, l}, 4 \widetilde{\delta}_{l}\left|w_{k, l}\right|\right)\right)$ satisfying the inequality

$$
\ln \left|f_{i}(z) / q_{4 \delta_{l}}\left(z, z_{k, l}, \Lambda\right)\right|-h_{f_{i}}(z)>-2 \varepsilon|z|,
$$

(respectively, the inequality

$$
\left.\ln \left|f_{i}(z) / q_{8 \widetilde{\delta}_{l}}\left(z, w_{k, l}, \Lambda\right)\right|>-2 \Theta|z|\right),
$$

where $\Lambda=\left\{\lambda_{j}\right\}$ is the set of all common zeros of $f_{1}, \ldots, f_{n}$. The specific choice of $\delta_{l}$ (respectively, $\widetilde{\delta}_{l}$ and $A_{l}$ ) and Definition 1 (respectively, Definition 4) allows us to conclude that the sets $\bigcup_{i=1}^{n} U_{k, l}^{i}$ (respectively, $\left.\bigcup_{i=1}^{n} \widetilde{U}_{k, l}^{i}\right)$ and $B\left(z_{k, l}, 2 \delta_{l}\left|z_{k, l}\right|\right.$ ) (respectively, $\left.B\left(w_{k, l}, 4 \widetilde{\delta}_{l}\left|w_{k, l}\right|\right)\right)$ coincide. Let $R_{0} \geq \max \left\{R_{l}, T_{p}: l=1, \ldots, l_{0} ; p=1, \ldots, l_{1}\right\}$. By construction, the union of all disks $B\left(z_{k, l}, 2 \delta_{l}\left|z_{k, l}\right|\right)$ and $B\left(w_{k, l}, 4 \widetilde{\delta}_{l}\left|w_{k, l}\right|\right)$ contains the set $\mathbb{C} \backslash B\left(0, R_{0}\right)$. Consequently, any point $z \in \mathbb{C}$ with $|z| \geq R_{0}$ belongs to at least one of the sets $U_{k, l}^{i}$ or $\widetilde{U}_{k, l}^{i}$. Let $x_{0}$ be an arbitrary point in $\mathbf{S} \subset B\left(0, R_{0}\right)$ that coincides with none of the common zeros $\lambda_{j}$ (we assume that $R_{0}>1$ ). Obviously, there is $\Theta^{\prime}>0$ such that

$$
\max _{1 \leq i \leq n} \ln \left|f_{i}(z) / q_{8 R_{0}}\left(z, x_{0}, \Lambda\right)\right|>-\Theta^{\prime}|z|, \quad z \in B\left(0,4 R_{0}\right) .
$$

For $i=1, \ldots, n$, let $U_{0}^{i}$ denote the set of all points $z \in B\left(0,4 R_{0}\right)$ such that

$$
\ln \left|f_{i}(z) / q_{8 R_{0}}\left(z, x_{0}, \Lambda\right)\right|>-2 \Theta^{\prime}|z| .
$$


Then, as above, the disk $B\left(0,4 R_{0}\right)$ coincides with $\bigcup_{i=1}^{n} U_{0}^{i}$. We put

$$
V_{i}=U_{0}^{i} \cup\left[\bigcup_{k=1}^{\infty} \bigcup_{l=1}^{l_{0}} U_{k, l}^{i}\right] \cup\left[\bigcup_{k=1}^{\infty} \bigcup_{l=1}^{l_{1}} \widetilde{U}_{k, l}^{i}\right] .
$$

Thus, we have constructed an open covering of the complex plane by the sets $V_{1}, \ldots, V_{n}$. This covering is determined by $\varepsilon>0$, by functions $f_{1}, \ldots, f_{n}$ satisfying conditions i) and ii), and by $Z \subset \mathbf{S}$. We denote

$$
X=\mathbf{S} \cap\left[\bigcup_{l=1}^{l_{1}} B\left(x_{l}, 4 \widetilde{\delta}_{l}\right)\right],
$$

where $\widetilde{\delta}_{l}$ and $x_{l}$ are the same as before.

Lemma 3.2. There exists a partition of unity $E_{i} \in C^{\infty}(\mathbb{C}), i=1, \ldots, n$, subordinate to the covering $V_{1}, \ldots, V_{n}$ of $\mathbb{C}$ (i.e., $E_{i}$ vanishes near the boundary of $V_{i}$ and off $V_{i}$ ) and such that the following inequalities are true for some $\widetilde{c}, \widetilde{b}>0$ :

$$
\left|d E_{i}(z) / d \bar{z}\right| \leq\left\{\begin{array}{ll}
\widetilde{c} \exp [12 \varepsilon|z|] & \text { if } z /|z| \notin X, \\
\widetilde{c} \exp [\widetilde{b}|z|] & \text { if } z /|z| \in X,
\end{array} \quad i=1, \ldots, n .\right.
$$

For the sequence $\Lambda=\left\{\lambda_{j}\right\}$ of common zeros (with regard to multiplicity) of functions $f_{1}, \ldots, f_{N}$ of exponential type, we define a function $f(z)$ by the formula

$$
f(z)=z^{m_{0}} \prod_{j=1, \lambda_{j} \neq 0}^{N}\left(1-\frac{z}{\lambda_{j}}\right) \exp \left(\frac{z}{\lambda_{j}}\right), \quad z \in \mathbb{C},
$$

where $m_{0}$ is the multiplicity of the common zero $z=0$ of the functions $f_{1}, \ldots, f_{N}$ (possibly, $m_{0}=0$ ). Since the points $\lambda_{j}, j=1,2, \ldots$, are zeros (part of the zeros) of an entire function (say, $f_{1}$ ) of order (at most) $\rho$ and of finite type (with order $\rho$ ), the above product converges everywhere on $\mathbb{C}$ and yields an entire function (see [2]). Moreover, $f(z)$ vanishes only at the points $\lambda_{j}$. For all $k=1,2, \ldots$ and $l=1, \ldots, l_{0}$, we put

$$
\psi_{k, l}(z)= \begin{cases}\ln |f(z)| & \text { if } z \notin B\left(z_{k, l}, 8 \delta_{l}\left|z_{k, l}\right|\right), \\ \max \left\{\ln |f(z)|, \ln \left|\frac{f(z)}{q_{4 \delta_{l}}\left(z, z_{k, l}, \Lambda\right)}\right|\right\} & \text { if } z \in B\left(z_{k, l}, 8 \delta_{l}\left|z_{k, l}\right|\right) .\end{cases}
$$

Also for $k=1,2, \ldots$ and $l=1, \ldots, l_{1}$, we introduce the functions $\widetilde{\psi}_{k, l}(z)$ by a similar formula, but with $B\left(z_{k, l}, 8 \delta_{l}\left|z_{k, l}\right|\right)$ and $q_{4 \delta_{l}}\left(z, z_{k, l}, \Lambda\right)$ replaced by $B\left(w_{k, l}, 16 \widetilde{\delta}_{l}\left|w_{k, l}\right|\right)$ and $q_{8 \widetilde{\delta}_{l}}\left(z, w_{k, l}, \Lambda\right)$, respectively. Finally, we put

$$
\psi_{0}(z)= \begin{cases}\ln |f(z)| & \text { if } z \notin B\left(x_{0}, 16 R_{0}\right), \\ \max \left\{\ln |f(z)|, \ln \left|f(z) / q_{8 R_{0}}\left(z, x_{0}, \Lambda\right)\right|\right\} & \text { if } z \in B\left(x_{0}, 16 R_{0}\right) .\end{cases}
$$

The above functions $\psi_{k, l}, \widetilde{\psi}_{k, l}$, and $\psi_{0}$ were constructed earlier in the paper [5], where it was shown that they are subharmonic in $\mathbb{C}$. Now we define

$$
\psi(z)=\sup \left\{\psi_{0}(z), \psi_{k, l}(z), \widetilde{\psi}_{p, r}\right\}, \quad z \in \mathbb{C},
$$

where the supremum is taken over all values of the indices $k, l, p, r$. The function $\psi(z)$ is subharmonic in $\mathbb{C}$, because near every point $z \in \mathbb{C}$ it is in fact the maximum of only finitely many subharmonic functions. The other functions coincide there (some neighborhood of every point $z \in \mathbb{C}$ intersects only finitely many disks $B\left(z_{k, l}, 8 \delta_{l}\left|z_{k, l}\right|\right)$ and $\left.B\left(w_{k, l}, 16 \widetilde{\delta}_{l}\left|w_{k, l}\right|\right)\right)$. 
Lemma 3.3. There exists a number $C>0$ such that the inequality

$$
\ln \left|f_{i}(z) / f(z)\right|+\psi(z) \leq C+H_{K_{i}}(z)+8 \varepsilon|z|, \quad z \in \mathbb{C},
$$

is fulfilled for all $i=1, \ldots, n$.

Now everything is ready for the discussion of conditions sufficient for solvability.

Theorem 3.4. Let the domains $D, G, G_{i}$, the compact sets $K_{i}$, the operators $M_{i}$, and the functions $f_{i}$ be the same as in the Introduction. Suppose that the following conditions are satisfied:

1) the system $\left(f_{1}, \ldots, f_{n}\right)$ has regular growth on each ray $t z, t>0$, with $z$ in the closure of the set $\mathbf{S} \backslash I_{G}$;

2) the system $\left(f_{1}, \ldots, f_{n}\right)$ has weak regular growth on each ray $t z, t>0$, with $z \in \mathbf{S} \cap I_{G}$.

Then the system of convolution equations

$$
M_{1}[h]=g_{1}, \ldots, \quad M_{n}[h]=g_{n}
$$

is solvable in the space $H(D)$ for any admissible right-hand side $\left(g_{1}, \ldots, g_{n}\right) \in H$.

Proof. By Lemma 1.2, it suffices to show that for any $F \in I(D, \Lambda)$ there exists an element $\left(\varphi_{1}, \ldots, \varphi_{n}\right)$ of the $\operatorname{space} P_{G_{1}} \times \cdots \times P_{G_{n}}$ such that

$$
F(z) \equiv \varphi_{1}(z) f_{1}(z)+\cdots+\varphi_{n}(z) f_{n}(z)
$$

for all $z \in \mathbb{C}$.

First, we establish some auxiliary inequalities. Since $F$ belongs to $I(D, \Lambda)$, and hence to $P_{D}$, the definition of the latter set shows that there is a compact set $K \subset D$ and a number $\widetilde{A}$ such that

$$
F(z) \leq \widetilde{A} \exp \left[H_{K}(z)\right], \quad z \in \mathbb{C} .
$$

The domain $D$ is of the form $D=G+K_{1}+\cdots+K_{n}$. It is easily seen that there exists a compact convex set $K_{0} \subset G$ such that $K \subset K_{0}+K_{1}+\cdots+K_{n}$. It follows that the support function of $K$ does not exceed the support function of the sum $K_{0}+K_{1}+\cdots+K_{n}$. Recalling that the support function of a sum of convex sets is equal to the sum of their support functions, we obtain

$$
|F(z)| \leq \widetilde{A} \exp \left[H_{K_{0}}(z)+H_{K_{1}}(z)+\cdots+H_{K_{n}}(z)\right], \quad z \in \mathbb{C} .
$$

Since some enlargement of the compact set $K$ will still lie in $G$, we can find $\varepsilon>0$ such that

$$
H_{K_{0}}(z)+32 \varepsilon|z|<H_{G}(z), \quad z \neq 0 .
$$

The function $H_{K_{0}}$ is uniformly continuous on the circle $\mathbf{S}$. Since it is homogeneous, there is $\delta_{0}>0$ such that $16 \delta_{0}<1$ and

$$
\left|H_{K_{0}}(z)-H_{K_{0}}(y)\right|<\varepsilon, \quad y \in B\left(z, 16 \delta_{0}\right), \quad z \in \mathbf{S} .
$$

Let $Z$ denote the closure of the set $\mathbf{S} \backslash I_{G}$. For $\varepsilon>0$ chosen as above, and for the set $Z$, we consider the covering $V_{1}, \ldots, V_{n}$ of $\mathbb{C}$ constructed as described before Lemma 3.2. We can construct this covering indeed, because the assumptions of the theorem imply that the functions $f_{1}, \ldots, f_{n}$ satisfy conditions i) and ii). Moreover, we may assume that the numbers $\delta_{l}$ and $\widetilde{\delta}_{p}$ occurring in the construction of that covering satisfy the inequality

$$
\delta_{l}, \widetilde{\delta}_{p}<\delta_{0}, \quad l=1, \ldots, l_{0}, \quad p=1, \ldots, l_{1} .
$$

For each $i=1, \ldots, n$, we seek the functions $\varphi_{i}(z)$ in the form

$$
\varphi_{i}=\frac{E_{i} F}{f_{i}}+\sum_{j=1}^{n} v_{i j} \frac{f_{j}}{f}
$$


(see [6. Appendix]), where $E_{1}, \ldots, E_{n}$ are the functions occurring in Lemma 3.2 and constructed starting with the covering $V_{1}, \ldots, V_{n}$ of $\mathbb{C}$, and the $v_{i j}$ are unknown functions, on which we impose the requirements $v_{i j}=-v_{j i}, v_{i i} \equiv 0$, in order that

$$
f_{1} \varphi_{1}+\cdots+f_{n} \varphi_{n} \equiv F
$$

automatically.

For the analyticity of $\varphi_{i}$ we need the identity $d \varphi_{i} / d \bar{z} \equiv 0$, which means that the functions $v_{i j}$ must satisfy the condition

$$
\frac{d E_{i}}{d \bar{z}} \frac{F}{f_{i}}+\sum_{j=1}^{n} \frac{d v_{i j}}{d \bar{z}} \frac{f_{j}}{f}=0
$$

This condition will be fulfilled if, for instance,

$$
\frac{d v_{i j}}{d \bar{z}}=\frac{E_{i} F f}{f_{i} f_{j}} \frac{d E_{j}}{d \bar{z}}-\frac{E_{j} F f}{f_{i} f_{j}} \frac{d E_{i}}{d \bar{z}}
$$

(this can be checked easily by using the identities $E_{1}+\cdots+E_{n} \equiv 1, \frac{d E_{1}}{d \bar{z}}+\cdots+\frac{d E_{n}}{d \bar{z}} \equiv 0$ ). First, we solve each of the equations

$$
\frac{d w_{i j}}{d \bar{z}}=\frac{E_{i} F f}{f_{i} f_{j}} \frac{d E_{j}}{d \bar{z}}
$$

and then put $v_{i j}=w_{i j}-w_{j i}$, so that $v_{i j}=-v_{j i}$ automatically.

Let a point $z \notin B\left(0, R_{0}\right)$ be such that $z /|z| \notin X$, where $X$ is the set defined before Lemma 3.2. Then $z \in B\left(z_{k, l}, 2 \delta_{l}\left|z_{k, l}\right|\right)$ for some $k$ and $l$. (Here we use the same notation as in the construction of $\left.V_{1}, \ldots, V_{n}.\right)$ By (2.2) and (3.2), we have

$$
\left|\frac{F(y)}{q_{4 \delta_{l}}\left(y, z_{k, l}, \Lambda\right)}\right| \leq|F(y)| \leq \widetilde{A} \exp \left\{\sum_{j=0}^{n} H_{K_{j}}(y)\right\}, \quad y \in \partial B\left(z_{k, l}, 8 \delta_{l}\left|z_{k, l}\right|\right) .
$$

The number $\delta_{l}$ satisfies $16 \delta_{l}<1$. Consequently, $B\left(z_{k, l}, 8 \delta_{l}\left|z_{k, l}\right|\right) \subset B\left(z, 16 \delta_{l}|z|\right)$. Using (3.1) (recall that $h_{f_{i}} \equiv H_{K_{i}}$ ), we deduce the inequality

$$
\left|\frac{F(y)}{q_{4 \delta_{l}}\left(y, z_{k, l}, \Lambda\right)}\right| \leq \widetilde{A} \exp \left\{\sum_{j=0}^{n} H_{K_{j}}(z)+2 \varepsilon|z|\right\}, \quad y \in \partial B\left(z_{k, l}, 8 \delta_{l}\left|z_{k, l}\right|\right) .
$$

Since $F \in I(D, \Lambda)$, the definitions of $I(D, \Lambda)$ and of the function $q_{4}\left(y, z_{k, l}, \Lambda\right)$ show that the ratio $F(y) / q_{4 \delta_{l}}\left(y, z_{k, l}, \Lambda\right)$ is an analytic (and even entire) function. From the maximum principle, it follows that the last-written inequality is valid everywhere in the disk $B\left(z_{k, l}, 8 \delta_{l}\left|z_{k, l}\right|\right)$ and, in particular, at the point $z$.

Recalling the definitions of the functions $\psi$ and $f$, of the sets $V_{1}, \ldots, V_{n}$, and of the functions $E_{1}, \ldots, E_{n}$ constructed as in Lemma 3.2 from the sets $V_{1}, \ldots, V_{n}$, we obtain

$$
\begin{array}{r}
\ln \left|\frac{d w_{i j}(z)}{d \bar{z}}\right| \leq \ln \left|\frac{E_{i}(z) \frac{F(z)}{q_{4 \delta_{l}}\left(z, z_{k, l}, \Lambda\right)} \frac{f(z)}{q_{4 \delta_{l}}\left(z, z_{k, l}, \Lambda\right)}}{\frac{f_{i}(z)}{q_{4 \delta_{l}}\left(z, z_{k, l}, \Lambda\right)} \frac{f_{j}(z)}{q_{4 \delta_{l}}\left(z, z_{k, l}, \Lambda\right)}} \frac{d E_{j}}{d \bar{z}}\right| \\
\leq \psi(z)+\sum_{p=0, p \neq i, j}^{n} H_{K_{p}}(z)+18 \varepsilon|z|+\ln \widetilde{A}+\ln \widetilde{c}
\end{array}
$$

where $\widetilde{c}$ is the same constant as in Lemma 3.2. In a similar way, we can prove that for the points $z /|z| \in X$ with $z \notin B\left(0, R_{0}\right)$ there exist numbers $\widetilde{A}_{1}$ and $B>0$ independent of $z$ such that

$$
\ln \left|\frac{d w_{i j}(z)}{d \bar{z}}\right| \leq \psi(z)+\sum_{p=1, p \neq i, j}^{n} H_{K_{p}}(z)+B|z|+\widetilde{A}_{1} .
$$


Unlike the preceding inequality, here summation starts with $p=1$, because the function $H_{K_{0}}$ is of no interest for us in this case, and its growth is compensated by the quantity $B$.

Now we need to unite the last two inequalities into a single integral estimate. For this, we use Lemma 3.1 to construct a compact set $L$ with certain properties. The roles of the domain, the compact set, and the number $a>0$ occurring in Lemma 3.1 will be played by the domain $G$, the closure of the set $K_{0}+B(0,19 \varepsilon)$ (by $(3.3)$, this closure lies in $G$ ), and the number $\varepsilon$, respectively. We verify the conditions of Lemma 3.1. The estimate assumed in Lemma 3.1 follows from (3.3). By construction, the set $X$ is compactly contained in the angle spanned by $\mathbf{S} \backslash Z$, and the role of $Z$ is played by the closure of $\mathbf{S} \backslash I_{G}$. Consequently, $X$ is compactly contained in int $I_{G}$. Thus, Lemma 3.1 is applicable. Therefore, for the number $A=B+\varepsilon$, in the domain $G$ we can find a compact convex subset $L$ containing $K_{0}+B(0,19 \varepsilon)$ and such that

$$
H_{L}(z)+11 \varepsilon|z|<H_{G}(z), \quad z \neq 0, \quad H_{L}(z)>(B+\varepsilon)|z| \text { if } z /|z| \in X .
$$

The inclusion $K_{0}+B(0,19 \varepsilon) \subset L$ means that

$$
H_{K_{0}}(z)+19 \varepsilon|z| \leq H_{L}(z), \quad z \in \mathbb{C} .
$$

Combining (3.7) and the above inequalities, we see that the estimate

$$
\left|\frac{d w_{i j}(z)}{d \bar{z}}\right| \leq \widetilde{A}_{2} \exp \left[\psi(z)+H_{L}(z)+\sum_{p=1, p \neq i, j}^{n} H_{K_{p}}(z)-\varepsilon|z|\right]
$$

is valid for all $z \in \mathbb{C} \backslash B\left(0, R_{0}\right)$. Observe that the derivative $d w_{i j}(z) / d \bar{z}$ has no singularities for any $z \in \mathbb{C}$. This follows from the definition of the $E_{i}$ and the fact that the functions $F, f, f_{i}$, and $f_{j}$ have a common zero set. Therefore, this derivative is bounded on $B\left(0, R_{0}\right)$. This allows us to assume that the above estimate is true at every point. Then the integral

$$
\int_{\mathbb{C}}\left|\frac{d w_{i j}(z)}{d \bar{z}}\right|^{2} \exp \left\{-2\left[\psi(z)+H_{L}(z)+\sum_{p=1, p \neq i, j}^{n} H_{K_{p}}(z)\right]\right\} d \sigma
$$

is finite. By [11, Theorem 4.4.2], it follows that equation (3.6) has a $C^{\infty}(\mathbb{C})$-solution $w_{i j}$ satisfying

$$
\int_{\mathbb{C}}\left|w_{i j}(z)\right|^{2} \exp \left\{-2\left[\psi(z)+H_{L^{\prime}}(z)+\sum_{p=1, p \neq i, j}^{n} H_{K_{p}}(z)\right]\right\} d \sigma<\infty,
$$

where $L^{\prime}$ is the closure of the set $L+B(0, \varepsilon)$. The Cauchy-Bunyakovskii inequality for integrals yields

$$
\int_{\mathbb{C}}\left|v_{i j}(z)\right|^{2} \exp \left\{-2\left[\psi(z)+H_{L^{\prime}}(z)+\sum_{p=1, p \neq i, j}^{n} H_{K_{p}}(z)\right]\right\} d \sigma<\infty .
$$

This inequality and Lemma 3.3 imply

$$
\int_{\mathbb{C}}\left|v_{i j}(z) \frac{f_{j}(z)}{f(z)}\right|^{2} \exp \left\{-2\left[H_{L^{\prime}}(z)+\sum_{p=1, p \neq i}^{n} H_{K_{p}}(z)+8 \varepsilon|z|\right]\right\} d \sigma<\infty .
$$

In a similar way we check that

$$
\int_{\mathbb{C}}\left|\frac{E_{i}(z) F(z)}{f_{i}(z)}\right|^{2} \exp \left\{-2\left[H_{L^{\prime}}(z)+\sum_{p=1, p \neq i}^{n} H_{K_{p}}(z)+8 \varepsilon|z|\right]\right\} d \sigma<\infty .
$$

This results in the estimate

$$
\int_{\mathbb{C}}\left|\varphi_{i}(z)\right|^{2} \exp \left\{-2\left[H_{L^{\prime}}(z)+\sum_{p=1, p \neq i}^{n} H_{K_{p}}(z)+8 \varepsilon|z|\right]\right\} d \sigma<\infty .
$$


Using the mean value inequality for subharmonic functions and the Cauchy-Bunyakovskii inequality for integrals, we obtain

$$
\begin{aligned}
\left|\varphi_{i}(y)\right| \leq & c \int_{B(y, 1)}\left|\varphi_{i}(z)\right| d \sigma \\
\leq & c\left[\int_{B(y, 1)}\left|\varphi_{i}(z)\right|^{2} \exp \left\{-2\left[H_{L^{\prime}}(z)+\sum_{p=1, p \neq i}^{n} H_{K_{p}}(z)+8 \varepsilon|z|\right]\right\} d \sigma\right]^{\frac{1}{2}} \\
& \times\left[\int_{B(y, 1)} \exp \left\{2\left[H_{L^{\prime}}(z)+\sum_{p=1, p \neq i}^{n} H_{K_{p}}(z)+8 \varepsilon|z|\right]\right\} d \sigma\right]^{\frac{1}{2}} \\
\leq & c_{1} \sup _{B(y, 1)} \exp \left\{H_{L^{\prime}}(z)+\sum_{p=1, p \neq i}^{n} H_{K_{p}}(z)+8 \varepsilon|z|\right\}
\end{aligned}
$$

where $c_{1}$ is independent of $y \in \mathbb{C}$. Now, since the support functions are uniformly continuous on the circle $\mathbf{S}$ and positively homogeneous on $\mathbb{C}$, it is not hard to deduce the estimate

$$
\left|\varphi_{i}(y)\right| \leq c_{2} \exp \left\{H_{L^{\prime}}(y)+\sum_{p=1, p \neq i}^{n} H_{K_{p}}(y)+9 \varepsilon|y|\right\}
$$

with $c_{2}>0$ independent of $y$. We recall that the compact set $L^{\prime}$ is the closure of $L+B(0, \varepsilon)$. Therefore, by 3.3 ,

$$
H_{L^{\prime}}(z)+\sum_{p=1, p \neq i}^{n} H_{K_{p}}(z)+9 \varepsilon|z| \leq H_{G}(z)-\varepsilon|z|+\sum_{p=1, p \neq i}^{n} H_{K_{p}}(z)=H_{G_{i}}(z)-\varepsilon|z|
$$

for all $z$. This means that the compact convex set $T$ coinciding with the $9 \varepsilon$-dilation of the set $L^{\prime}+\sum_{p=1, p \neq i}^{n} K_{p}$ is contained in $G_{i}$. By the facts proved above, we have

$$
\left|\varphi_{i}(z)\right| \leq c_{2} \exp H_{T}(z), \quad z \in \mathbb{C} .
$$

Consequently, $\varphi_{i} \in P_{G_{i}}, i=1, \ldots, n$. The theorem is proved.

In the case where $D$ is a domain with smooth boundary, the regular growth conditions are also necessary. The following statement is true.

Theorem 3.5. Let $G$ be a domain with smooth boundary and such that $I_{G}$ is an open (possibly, empty) set. Then system (1.3) is solvable in $H(D)$ for any admissible righthand side of class $H$ if and only if the following conditions are satisfied:

1) the system of functions $\left(f_{1}, \ldots, f_{n}\right)$ has regular growth on each ray $t \eta$ with $\eta \in \mathbf{S} \backslash I_{G}$;

$2)$ the system of functions $\left(f_{1}, \ldots, f_{n}\right)$ has weak regular growth on each ray t $\eta$ with $\eta \in \mathbf{S} \cap I_{G}$.

Proof. The "if" part follows from Theorem 3.4 The "only if" part is a consequence of 5. Propositions 4.3 and 4.4].

\section{REFERENCES}

[1] V. V. Napalkov, Convolution equations in multidimensional spaces, "Nauka", Moscow, 1982. (Russian) MR0678923 (86g:46054)

[2] B. Ya. Levin, Distribution of zeros of entire functions, Gostekhizdat, Moscow, 1956; English transl., Amer. Math. Soc., Providence, RI, 1964. MR0087740 (19:402c) MR0589888 (81k:30011)

[3] A. S. Krivosheev, A criterion for the solvability of inhomogeneous convolution equations in convex domains of the space $\mathbb{C}^{n}$, Izv. Akad. Nauk. SSSR Ser. Mat. 54 (1990), no. 3, 480-500; English transl., Math. USSR-Izv. 36 (1991), no. 3, 497-517. MR1072692|(91k:46037) 
[4] A. S. Krivosheev and V. V. Napalkov, Complex analysis and convolution operators, Uspekhi Mat. Nauk 47 (1992), no. 6, 3-58; English transl., Russian Math. Surveys 47 (1992), no. 6, 1-56. MR.1209144(94e:32003)

[5] A. S. Krivosheev, Regularity of the growth of a system of functions and a system of nonhomogeneous convolution equations in convex domains of the complex plane, Izv. Ross. Akad. Nauk Ser. Mat. 64 (2000), no. 5, 69-132; English transl., Izv. Math. 64 (2000), no. 5, 939-1001. MR1789187 (2001m:46046)

[6] P. Koosis, Introduction to $H^{p}$ spaces. With an appendix on Wolff's proof of the corona theorem, London Math. Soc. Lecture Note Ser., vol. 40, Cambridge Univ. Press, Cambridge-New York, 1980. MR.0565451 (81c:30062)

[7] J. Dieudonné and L. Schwartz, La dualité dans les espaces $(F)$ et $(L F)$, Ann. Inst. Fourier (Grenoble) 1 (1950), 61-101. MR0038553(12:417d)

[8] J. J. Wiegerink, Growth properties of Paley-Wiener functions on $\mathbb{C}^{n}$, Nederl. Akad. Wetensch. Indag. Math. 46 (1984), 95-112. MR.0748983 (86b:32002)

[9] R. Segurdsson, Convolution equations in domains of $\mathbb{C}^{n}$, Ark. Mat. 29 (1991), 285-305. MR1150379 (93b:46074)

[10] A. F. Leont'ev, Entire functions. Series of exponentials, "Nauka", Moscow, 1983. (Russian) MR.0753827 (86j:30005)

[11] L. Hörmander, The analysis of linear partial differential operators. I. Distribution theory and Fourier analysis, 2nd ed., Grundlehren Math. Wiss., vol. 256, Springer-Verlag, Berlin, 1990. MR:1065136 (91m:35001b)

Received 17/APR/2002

Translated by A. PLOTKIN 